\title{
Performance Analysis of Multicell Multiuser MIMO Precoding Using Partial Knowledge at the Transmitter
}

\author{
Lígia Maria Carvalho Sousa and Charles Casimiro Cavalcante
}

\begin{abstract}
Resumo-A disponibilidade da informação do estado do canal no transmissor (Channel State Information at the Transmitter - CSIT) melhora o desempenho dos sistemas sem fio MIMO multiusuário através do uso de técnicas de precodificação. A maioria dessas técnicas assume que o transmissor conhece o canal perfeitamente. Um CSIT preciso pode ser obtido de forma confiável quando o canal muda lentamente; contudo, é muito difícil obter tal informação quando os usuários se movimentam rapidamente. Dessa forma, em tais cenários, CSIT imperfeito ou parcial tem uma grande importância. Neste trabalho apresentamos um modelo de canal dinâmico multiusuário que considera processamento multicelular entre as BSs, no qual cada BS transmite para todos os usuários. Este cenário é bastante visado para o gerenciamento de interferência em redes sem fio das próximas gerações.
\end{abstract}

Palavras-Chave-CSIT parcial, precodificação, canal de retorno restrito, sistema MIMO multiusuário, antenas distribuídas.

Abstract-Channel state information at the transmitter (CSIT) can improve the performance of MIMO multiuser wireless system by means of precoding techniques. Most of them assume the transmitter knows the channel exactly. Accurate CSIT may be obtained reliably when the channel changes slowly, but it is much more difficult to detain in situations where the users are highly mobile. Thus, partial or imperfect CSIT has a great importance in such scenarios. In this work, we present a multiuser dynamic channel model considering multicell processing among BSs, in which each BS transmits to all users. This is the scenario envisaged for interference management in future wireless networks.

Keywords - Partial CSIT, precoding, limited feedback, multiuser MIMO system, distributed antennas.

\section{INTRODUCTION}

Multiple-input multiple-output (MIMO) systems are a key component of future wireless communication systems, because of their promising improvement in terms of performance and bandwidth efficiency. Distributed antenna systems (DAS) is a network of spatially separated antenna nodes connected to a common source via a transport medium that provides wireless service within a geographic area or structure [1].

The benefits of MIMO are further enhanced when the transmitter, in addition to the receiver, also knows the communication channel. Exploiting channel state information at the transmitter (CSIT) is of great practical interest in MIMO wireless systems, since it helps increase the system capacity and improve the system error performance. It has application

This work was supported by the Research and Development Center Ericsson Telecommunicações S.A., Brazil, under EDB/UFC.25 Technical Cooperation Contract. Federal University of Ceará (UFC). Department of Teleinformatic Engineering (DETI). Email: \{ligia, charles\}@gtel.ufc.br in areas such as precoding [2], power control [3] and link adaptation [4]. Precoding is a processing technique that exploits CSIT by operating on the signal before transmission. Most of precoding designs rely on the assumption that the channel is perfectly known at the transmitter [5] [6]. However, it is not reasonable to assume that all channel coefficients to every user can be made available to the transmitter. This is especially true if the number of transmit antennas or the number of users is large.

In this work, a multicell multiuser dynamic model channel is proposed and well-known precoder techniques are used to valid the proposed model. Moreover, we consider a MIMO system with a multicell processing scheme, in which each BS transmits to all users.

The rest of the paper is organized as follows. In section II, the multicell multiuser MIMO system considering DAS is commented. In section III, the proposed channel model is presented. Section IV explains about the power allocation employed and the precoding techniques simulated. Simulation results are in Section V. Finally, conclusions and perspectives are stated in Section VI.

\section{Multicell Multiuser System Model}

Distributed antenna systems (DAS) is a new architecture for future public wireless access, which refers to a generalized MIMO system comprising an antenna array at one side of the link and several largely separated antenna arrays at the other side [1]. The main advantage of DAS is the macrodiversity, which is inherent to the widely spaced antennas and offers the capability to enhance signal quality, increase system capacity and improve coverage.

In this work, we will consider a multiuser MIMO system composed by three cells, in which the BSs process signals jointly in order to enhance the system performance. This is called multicell processing (MCP) and is an inherent feature of DAS. Some important characteristics in this model must be elaborated on:

1) The channel gains between any user and the distributed antennas of the BSs may have different statistics, as dictated by the characteristics of propagation, such as path loss, shadowing, line-of-sight components, and the system topology. Thus, the transmit power of BSs are different and power constraints have to be enforced on a per-BS basis;

2) The BSs are connected through high capacity links (referred to as the backbone) to a central processing 
unit, which jointly processes the signals. In this work, an ideal delayless, infinite-capacity backbone is assumed to connect all BSs to the central unit.

Consider $K$ cochannel mobile stations (MS) arbitrarily distributed in the multicell system, composed by $N_{b}$ cells whose BSs cooperate with each other. Each MS is equipped with $N_{r}$ receive antennas and each BS with $N_{t}$ transmit antennas. Hereafter $\left(N_{t}, N_{r}, N_{b}, K\right)$ will be used to represent the overall structure of the system. In this work, we consider that a scheduling algorithm selects the best user in each cell to transmit before of the precoding. Thus, the number of cells is equal to total number of users $\left(N_{b}=K\right)$. Figure 1 shows this representation for a case with $N_{b}=K=3$.

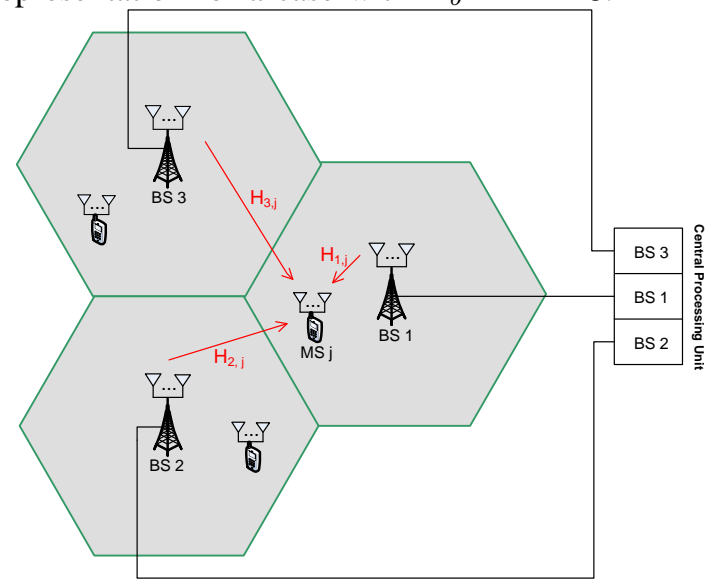

Fig. 1. Multicell multiuser MIMO system model with $N_{b}=K=3$.

The channel is considered frequency-flat and $\mathbf{H}_{b, j}$ is the $N_{r} \times N_{t}$ channel matrix seen by mobile station $j$ from base station $b$. It takes into account all signal losses: path-loss, shadowing and small-scale fading. The last one is modeled using Jake's model [7].

As already mentioned, in a MCP scenario, the transmit signal for each user is spread over all $N_{b}$ BSs. Thus, the signal vector intended for user $j$ can be expressed as $\mathbf{x}_{j}=\left[\mathbf{x}_{j}^{[1] T}, \mathbf{x}_{j}^{[2] T}, \ldots, \mathbf{x}_{j}^{\left[N_{b}\right] T}\right]_{N_{b} N_{t} \times 1}^{T}$ where $\mathbf{x}_{j}^{[b]} \in \mathbb{C}^{N_{t} \times 1}$ is the transmitted signal intended for user $j$ from BS $b$. It is assumed a perfect synchronism among the signals from different BSs to user $j$.

The received signal at MS $j$ is:

$$
\mathbf{y}_{j}=\mathbf{H}_{\Sigma j} \mathbf{x}_{j}+\sum_{k \neq j} \mathbf{H}_{\Sigma j} \mathbf{x}_{k}+\mathbf{n}_{j}
$$

where

$$
\mathbf{H}_{\Sigma j}=\left[\mathbf{H}_{1, j}, \mathbf{H}_{2, j}, \ldots, \mathbf{H}_{N_{b}, j}\right]_{N_{r} \times N_{b} N_{t}}
$$

is the joint channel matrix for user $j$ and $\mathbf{n}_{j}$ is the background noise.

Let $L_{j}$ denote the number of data streams intended for user $j$. For each MS $j(j=1,2, \ldots, K)$, an $N_{t} N_{b} \times L_{j}$ precoder matrix $\mathbf{T}_{j}$ is designed based on the characteristics of $\mathbf{H}_{\Sigma j}$. Thus, the transmitted signal for user $j$ is equal to $\mathbf{x}_{j}=\mathbf{T}_{j} \mathbf{s}_{j}$, where $\mathbf{s}_{j} \in \mathbb{C}^{L_{j} \times 1}$ corresponds to the substreams of the MS $j$.

Equation (1) can be rewritten as:

$$
\mathbf{y}_{j}=\mathbf{H}_{\Sigma j} \mathbf{T s}+\mathbf{n}_{j}
$$

where

$$
\mathbf{T}=\left[\mathbf{T}_{1}, \mathbf{T}_{2}, \ldots, \mathbf{T}_{K}\right]_{N_{t} N_{b} \times L_{T}}
$$

is the joint precoding matrix and

$$
\mathbf{s}=\left[\mathbf{s}_{1}^{T}, \ldots, \mathbf{s}_{j}^{T}, \ldots, \mathbf{s}_{K}^{T}\right]_{L_{T} \times 1}^{T}
$$

is the joint data streams vector and $L_{T}=\sum_{j=1}^{K} L_{j}$.

The streams, for simplicity, are assumed to have i.i.d. zeromean unit-variance complex Gaussian entries, i.e., Gaussian signalling assumption.

\section{PARTIAL CSIT}

A major challenge in wireless communication is the timevariation of the channel. This time-variation creates difficulty in obtaining channel information, which is required for best performance.

Most of precoding techniques relies on the fact that the transmitter knows perfectly the channel matrix [2] [5]. However, the random time-varying wireless medium makes difficult and often expensive to obtain perfect CSIT. Therefore, the transmitter has often only partial channel information and, thus, schemes exploiting partial CSIT are both important and necessary, and the obtained results are more modest. The first use of partial CSI at the transmitter was introduced in [8], where the Lloyd algorithm is used to quantize the CSI.

Some limited feedback multiuser MIMO schemes let users quantize some function of the channel matrix and send this channel information to the base station [9] [10]. The problem occurs when the user signals can not be perfectly orthogonalized by precoder due to channel quantization error. In order to avoid this error propagation, it is proposed schemes that directly select a quantized precoder from a codebook at the receiver, and feedback the precoder index to the transmitter [11] [12]. However, it is too difficult to design the precoder codebook.

Other approaches focused on mean matrix of the channel [13], or on covariance matrix of the channel as a form of feedback [14]. These information reveal a great deal about the slow fading and the mean separability of the users.

The channel model proposed in [15] takes into account channel time-variation. The model relies on stochastic processes and estimation theories. Derived from a potentially outdated channel measurement and the channel statistics (mean and covariance), this dynamic CSIT consists of a channel estimate and its error covariance, acting as the effective channel mean and covariance. Both parameters depend on a temporal correlation factor, indicating the CSIT quality. Depending on this quality, the model covers smoothly from perfect to statistical channel information. This dynamic CSIT is applicable to all Gaussian random channels, however it was only proposed for MIMO single user system. In next subsection, the dynamic CSIT is presented in a general multicell multiuser context.

\section{A. Proposal: The Multicell Multiuser Dynamic CSIT}

The joint channel matrix $\mathbf{H}$ can be decomposed as

$$
\mathbf{H}=\overline{\mathbf{H}}+\tilde{\mathbf{H}},
$$

where $\overline{\mathbf{H}}$ is the joint channel mean and $\tilde{\mathbf{H}}$ is the joint zeromean Gaussian component. 
The joint channel mean $\overline{\mathbf{H}}$ is defined as

$$
\begin{aligned}
\overline{\mathbf{H}} & =\left[\begin{array}{lll}
\overline{\mathbf{H}}_{\Sigma 1}^{T}, & \ldots, & \overline{\mathbf{H}}_{\Sigma K}^{T}
\end{array}\right]^{T} \\
= & \left.\mathbb{E}\left\{\begin{array}{lll}
\mathbf{H}_{\Sigma 1}^{T}, & \ldots, & \mathbf{H}_{\Sigma K}^{T}
\end{array}\right]^{T}\right\}
\end{aligned}
$$

and the joint channel covariance $\mathbf{R}_{\mathbf{0}}$ is defined as:

$$
\mathbf{R}_{\mathbf{0}}=\mathbb{E}\left\{\mathbf{h} \mathbf{h}^{H}\right\}-\overline{\mathbf{h}} \overline{\mathbf{h}}^{H}
$$

where

and $\mathbf{h}=\operatorname{vec}(\mathbf{H})$

$$
\mathbf{H}=\left[\begin{array}{lll}
\mathbf{H}_{\Sigma 1}^{T}, & \ldots, & \mathbf{H}_{\Sigma K}^{T}
\end{array}\right]^{T}
$$

In begin of the simulation, it is assumed that the central unit has a joint initial channel measurement $\mathbf{H}_{\mathbf{0}}$ at time 0 , together with the channel statistics $\overline{\mathbf{H}}$ and $\mathbf{R}_{\mathbf{0}}$. These statistical matrices are obtained by averaging instantaneous channel measurement over tens of coherence times and they remain valid for a period of tens to hundreds coherence times, during which, the channel is considered stationary.

However, it is not trivial to obtain these matrices in a multicell multiuser scenario. Assume a simplified scenario composed by 2 cells with 1 user in each cell. Each BS has 2 transmit antennas and each MS has 1 receive antenna. So, in the initial interval time, each MS $j$ will send to the BSs its corresponding channel mean matrix $\overline{\mathbf{H}}_{\Sigma j}$, channel covariance matrix $\mathbf{R}_{\Sigma j_{-} \mathbf{0}}$ and channel initial measurement matrix $\mathbf{H}_{\Sigma j_{-} \mathbf{0}}$ which are given by following equations (considering the user 1):

$$
\begin{aligned}
& \overline{\mathbf{H}}_{\Sigma 1}= {\left[\begin{array}{ll}
\overline{\mathbf{H}}_{1,1} & \overline{\mathbf{H}}_{2,1}
\end{array}\right] } \\
& \mathbf{R}_{\Sigma 1 \_0}= \mathbb{E}\left\{\mathbf{h}_{\Sigma 1} \mathbf{h}_{\Sigma 1}^{H}\right\} \\
& \mathbf{R}_{\Sigma 1 \_\mathbf{0}}=\left[\begin{array}{ll}
\mathbb{E}\left\{\mathbf{h}_{1,1} \mathbf{h}_{1,1}^{H}\right\} & \mathbb{E}\left\{\mathbf{h}_{1,1} \mathbf{h}_{2,1}^{H}\right\} \\
\mathbb{E}\left\{\mathbf{h}_{2,1} \mathbf{h}_{1,1}^{H}\right\} & \mathbb{E}\left\{\mathbf{h}_{2,1} \mathbf{h}_{2,1}^{H}\right\}
\end{array}\right] \\
& \mathbf{H}_{\Sigma 1 \_\mathbf{0}}=\left[\mathbf{H}_{1,1 \_\mathbf{0}} \mathbf{H}_{2,1 \_\mathbf{0}}\right]
\end{aligned}
$$

where $\mathbf{H}_{i, j}$ is the channel gain matrix from BS $i$ to MS $j$.

The BSs will send this information to the central unit and the central unit will form the joint channel mean matrix, the joint channel covariance and the joint initial measurement in following form:

$$
\begin{gathered}
\overline{\mathbf{H}}=\left[\begin{array}{l}
\overline{\mathbf{H}}_{\Sigma 1} \\
\overline{\mathbf{H}}_{\Sigma 2}
\end{array}\right]=\left[\begin{array}{ll}
\overline{\mathbf{H}}_{1,1} & \overline{\mathbf{H}}_{2,1} \\
\overline{\mathbf{H}}_{1,2} & \overline{\mathbf{H}}_{2,2}
\end{array}\right], \\
\mathbf{R}_{\mathbf{0}}=\mathbb{E}\left\{\mathbf{h h}^{H}\right\},
\end{gathered}
$$

where

$$
\mathbf{H}=\left[\begin{array}{l}
\mathbf{H}_{\Sigma 1} \\
\mathbf{H}_{\Sigma 2}
\end{array}\right]=\left[\begin{array}{llll}
H_{1,1}^{1,1} & H_{1,1}^{2,1} & H_{2,1}^{1,1} & H_{2,1}^{2,1} \\
H_{1,2}^{1,1} & H_{1,2}^{2,1} & H_{2,2}^{1,1} & H_{2,2}^{2,1}
\end{array}\right]
$$

and $H_{b, j}^{t, r}$ is the channel gain from transmit antenna $t$ of BS $b$ to receive antenna $r$ of MS $j$.

$$
\mathbf{H}_{\mathbf{0}}=\left[\begin{array}{l}
\mathbf{H}_{\mathbf{0} \_\Sigma 1} \\
\mathbf{H}_{\mathbf{0} \_\Sigma 2}
\end{array}\right]=\left[\begin{array}{ll}
\mathbf{H}_{\mathbf{0} \_1,1} & \mathbf{H}_{\mathbf{0} \_2,1} \\
\mathbf{H}_{\mathbf{0} \_2,1} & \mathbf{H}_{\mathbf{0} \_2,2}
\end{array}\right]
$$

Since these joint matrices are formed from the matrices sent by the users, it is possible to note that some elements of the joint covariance matrix (Equation (15)) will not be available in the central unit. Such elements of the joint covariance matrix are those that contain channel gains of different users, such as the channel elements $\mathbb{E}\left\{H_{1,1}^{1,1} H_{1,2}^{1,1 *}\right\}$ or $\mathbb{E}\left\{H_{2,1}^{1,1} H_{2,2}^{1,1 *}\right\}$. It is not possible for the central unit to calculate these elements from the covariance matrices sent by each user.

Thus, we suppose that the expectation of the product of these elements is equal to the product of the expectation of the each element, i.e.,

$$
\mathbb{E}\left\{H_{b 1, j 1}^{t 1, r 1} H_{b 2, j 2}^{t 2, r 2 *}\right\}=\mathbb{E}\left\{H_{b 1, j 1}^{t 1, r 1}\right\} \cdot \mathbb{E}\left\{H_{b 2, j 2}^{t 2, r 2 *}\right\} .
$$

This approximation is considered true since the channel gains of users in different cells can be considered independent. Also it is considered feasible since that the expectation of the each channel element is available in the central unit from the joint mean matrix $\overline{\mathbf{H}}$.

With the joint initial channel measurement $\mathbf{H}_{\mathbf{0}}$ at time 0 , together with the channel statistics $\overline{\mathbf{H}}$ and $\mathbf{R}_{\mathbf{0}}$ at the transmitter, the estimate of the current channel $\mathbf{H}_{n}$ at the transmit time $n$ is established.

The channel auto-covariance $\mathbf{R}_{\Sigma j \_n}$ of user $j$ at time $n$ captures both the channel temporal correlation and the antenna correlation; and correlates the initial channel measurement with the current channel:

$$
\mathbf{R}_{\Sigma j_{-} n}=\mathbb{E}\left\{\mathbf{h}_{\Sigma j \_n} \mathbf{h}_{\Sigma j_{-} \mathbf{0}}^{H}\right\}-\overline{\mathbf{h}}_{\Sigma \mathbf{j}} \overline{\mathbf{h}}_{\Sigma \mathbf{j}}^{H} .
$$

Assuming that the channel gains between all transmit antennas and the receive antennas of the same user $j$ have the same temporal correlation function at time $n \rho_{j \_n}$, it is then possible to separate the temporal correlation from the antenna correlation in the channel auto-covariance as

$$
\mathbf{R}_{\Sigma j \_n}=\rho_{j \_n} \mathbf{R}_{\Sigma j \_} \mathbf{0},
$$

where $\rho_{j_{n} n}$ is a function of the Doppler spread $f_{d_{j}}$ for user $j$ at time delay $n$. In Jake's model, the channel temporal variation is given by:

$$
\rho_{j \_n}=J_{0}\left(2 \pi n f_{d_{j}}\right),
$$

where $J_{0}($.$) is the zero-th order Bessel function of the first$ kind [7] [16].

An estimative of the channel at time $n$ together with the estimation error covariance can be obtained from MMSE estimation theory and the results are given as:

$$
\hat{\mathbf{H}}_{n}=\Delta_{n} \mathbf{H}_{0}+\left(\mathbf{1}-\Delta_{n}\right) \overline{\mathbf{H}}
$$

where

$$
\Delta_{n}=\left[\begin{array}{cccc}
\rho_{1 \_n} \mathbf{I}_{N_{r}} & 0 & \cdots & 0 \\
0 & \rho_{2 \_n} \mathbf{I}_{N_{r}} & \cdots & 0 \\
\vdots & \vdots & \ddots & \vdots \\
0 & 0 & \cdots & \rho_{K \_n} \mathbf{I}_{N_{r}}
\end{array}\right]_{K N_{r} \times K N_{r}}
$$

with $\mathbf{I}_{k}$ being the identity matrix of size $k \times k$. The error covariance matrix $\mathbf{R}_{e_{-} n}$ is:

$$
\mathbf{R}_{e_{-} n}=\left(\mathbf{1}_{N_{b} N_{t} K N_{r}}-\Lambda_{n}\right) \star \mathbf{R}_{0}
$$

where

$$
\Lambda_{n}=\mathbf{1}_{N_{b} N_{t}} \otimes\left(\Upsilon_{n} \otimes \mathbf{1}_{N_{r}}\right)
$$

and

$$
\Upsilon_{n}=\left[\begin{array}{cccc}
\rho_{1 \_n} \rho_{1 \_n} & \rho_{1 \_n} \rho_{2 \_n} & \cdots & \rho_{1 \_n} \rho_{K \_n} \\
\rho_{2 \_n} \rho_{1 \_n} & \rho_{2 \_n} \rho_{2 \_n} & \cdots & \rho_{2 \_n} \rho_{K \_n} \\
\vdots & \vdots & \ddots & \vdots \\
\rho_{K \_n} \rho_{1 \_n} & \rho_{K \_n} \rho_{2 \_n} & \cdots & \rho_{K \_n} \rho_{K \_n}
\end{array}\right]_{K \times K}
$$

with $\mathbf{1}_{k}$ being a matrix $k \times k$ composed by 1 s and the operation ( $\star$ meaning the point-to-point multiplication of the matrices. 
These two quantities $\hat{\mathbf{H}}_{n}$ and $\mathbf{R}_{e_{-} n}$ work effectively as a new channel mean and a new channel covariance, and thus are referred to as the effective mean and the effective covariance, respectively. Together, they constitute the CSIT. This CSIT ranges from perfect channel knowledge when $\rho=1$ to pure statistics when $\rho=0$. Since the CSIT depends on $\rho$ which captures the channel temporal variation, it is called dynamic CSIT.

Thus, the channel matrix at time instant $n$ is given by:

$$
\mathbf{H}_{n}=\hat{\mathbf{H}}_{n}+\mathbf{R}_{r, n}^{1 / 2} \mathbf{H}_{w} \mathbf{R}_{t, n}^{1 / 2},
$$

where $\mathbf{H}_{w}$ is a $K N_{r} \times N_{b} N_{t}$ joint channel matrix whose entries have the real and imaginary parts independent and identically distributed as zero-mean Gaussian with unit-variance. The matrices $\mathbf{R}_{t, n} \in \mathbb{C}^{N_{b} N_{t} \times N_{b} N_{t}}$ and $\mathbf{R}_{r, n} \in \mathbb{C}^{K N_{r} \times K N_{r}}$ are obtained by Kronecker decomposition of the $\mathbf{R}_{e, n}=$ $\mathbf{R}_{t, n}^{T} \otimes \mathbf{R}_{r, n}$.

In this work, we obtain the Kronecker decomposition by the minimization of the term $\left\|\mathbf{R}_{e, n}-\mathbf{R}_{t, n}^{T} \otimes \mathbf{R}_{r, n}\right\|_{\mathcal{F}}$ demonstrated in [17]. Here, $\mathbf{R}_{t, n}^{1 / 2}$ is the principal square-root of $\mathbf{R}_{t, n}$, such that, $\mathbf{R}_{t, n}^{1 / 2} \mathbf{R}_{t, n}^{1 / 2}=\mathbf{R}_{t, n}$; similarly for $\mathbf{R}_{r, n}$.

\section{MiMO MU PRECoding Techniques}

The necessity of the cheaper mobiles with low power consumption increases the focus on system where the computationally demanding signal processing is performed at the BS. Precoding is a processing technique that exploits the channel state information at the transmitter (CSIT) by operating on the signal before transmission in order to mitigate or eliminate the interference.

In the literature, there are various precoding techniques that can be divided into linear and non-linear cases. The linear precoding assumes that the transmitted signal is generated by a linear combination of input data symbols. Some examples of these techniques are zero-forcing (ZF) [16], minimum meansquare-error (MMSE) and block-diagonalization (BD) [18]. The non-linear precoding techniques are based on the concept of coding technique proposed by Costa known as "dirtypaper coding" (DPC) [19]. DPC techniques can achieve the maximum sum rate of the system and provide the maximum diversity order [20]. However, these techniques require the use of a complex sphere-encoder or an approximate closest-point solution, which makes them hard to implement in practice [21]. Moreover, non-linear MU MIMO precoding techniques require the instantaneous knowledge of the channel transfer function at the BS.

On the other hand, linear MU MIMO precoding techniques are less computationally demanding than DPC ones, and they can use either instantaneous channel knowledge or long-term statistics of the channel. Thus, such techniques are more flexible and more favorable for practical implementation than non-linear techniques.

Having per-base power constraints is an intrinsic characteristic of MCP. A suboptimal way of obtaining the precoding matrix $\mathbf{T}$, defined in equation (4), is to use the already known results for precoder techniques without considering their scale factors (which is the classical approach for global power constraints), and afterwards the per-base power constraints can be imposed by applying a power loading matrix. Thus, the matrix $\mathbf{T}$ can be seen as a product of two others matrices,

$$
\mathbf{T}=\mathbf{F} \Omega
$$

where $\mathbf{F}$ is the precoder matrix without any power loading. Such matrix is a collection of submatrices $\mathbf{F}_{j}$, grouped sideby-side,

$$
\mathbf{F}=\left[\mathbf{F}_{1}, \mathbf{F}_{2}, \ldots, \mathbf{F}_{K}\right]_{N_{t} N_{b} \times L_{T}}
$$

where $\mathbf{F}_{j}$ represents the precoding matrix for the user $j$. The matrix $\boldsymbol{\Omega}=\mu \mathbf{I}$ is an $L_{T} \times L_{T}$ diagonal matrix with $\mu$ being the power allocated equally for the original data streams. Let $\mathbf{P}_{T}=\left[P_{\mathrm{BS} \_1}, P_{\mathrm{BS} \_2}, \cdots, P_{\mathrm{BS} \_N_{b}}\right]^{T}$ be the per-base constraint vector. Then the matrix $\Omega$ can be calculated as:

$$
\boldsymbol{\Omega}=\mu \mathbf{I}, \quad \mu=\min _{b=1,2, \cdots, N_{B}} \sqrt{\left(\frac{P_{\mathrm{BS} \_b}}{\left\|\mathbf{F}^{[b]}\right\|_{\mathcal{F}}^{2}}\right)},
$$

where $\mathbf{F}^{[b]}$ contains the rows of $\mathbf{F}$ corresponding to the transmit antennas at BS $b$ [22] and $\|.\|_{\mathcal{F}}$ is the Frobenius norm.

The precoding matrix $\mathbf{F}$ is chosen according to existent precoding techniques known as Zero Forcing (ZF) [16], Minimum Mean Square Error (MMSE) [23] and Block-Diagonalization [18]. ZF precoding eliminates all interference at the user terminal, but suffers from the transmit signal attenuation. Thus it is sub-optimal approach and results in a significant performance degradation. MMSE precoder makes a tradeoff between interference cancelation and transmitter power efficiency. In the same way as the receive spatial MMSE filter, it approximates a matched filter at low SNRs and is near optimal. At high SNRs, the MMSE precoder converges to a $\mathrm{ZF}$ precoder. $\mathrm{BD}$ precoder is a $\mathrm{ZF}$ precoding technique that was proposed to solve either the problem of maximizing the total system throughput under a transmit power constraint or to minimize the total transmit power for a predefined QoS level [18].

\section{Simulations AND RESUlts}

The simulator follows a Monte-Carlo approach. It simulates the downlink of a multicell multiuser MIMO system, where base-stations can process jointly to precancel the effect of interference among cochannel users. The multiuser dynamic channel model is analyzed in such scenario.

The considered channel model is frequency-flat block fading, where in each block the channel varies following Jake's model. Thus, in each block, fast fading is present considering the Doppler spread effect. During the simulation, the channel is considered stationary during two thousand coherence times.

For simulation of the multiuser dynamic model, we have the following steps:

1) The users send to BSs the channel statistics (mean $\overline{\mathbf{H}}$ and covariance $\mathbf{R}_{0}$ ) at the beginning of simulation. Such matrices keep valid during all simulation, where the channel is considered stationary. (See Figure 2 )

2) At the beginning of each block fading, the users send the corresponding initial measurement channel $\mathbf{H}_{0 \_\Sigma j}$ to BS.

3) Since that $\rho_{\Delta t}=J_{0}\left(2 \pi \Delta t f_{d}\right)$, the duration of the blocks $T_{B}$ is equal to $T_{B}=\Delta t=\frac{\arg J_{0}\left(\rho_{\Delta t}\right)}{2 \pi f_{d}}$. Thus, for each $\rho_{\Delta t}$, the number of transmitted symbols per 
block changes. Table I shows the number of transmitted symbols per block $\left(N_{S}\right)$ for each $\rho_{\Delta t}$ adopted.

4) During this block, the channel is estimated using equations (21), (22) and (23).

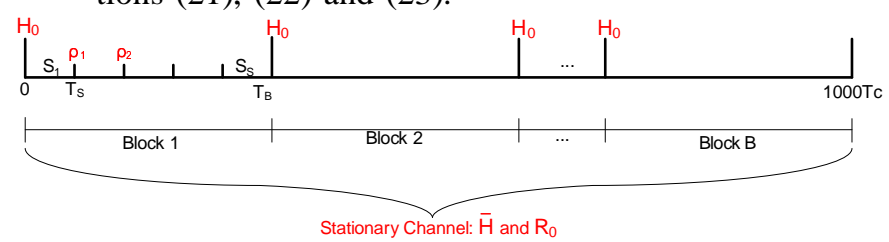

Fig. 2. Modeling of dynamic channel model.

TABLE I

EVALUation of the Number of TRANSMitTEd Symbols PER BLOCK

\begin{tabular}{r|c|c|c|}
\hline$\rho_{\triangle t}$ & $\arg J_{0}\left(\rho_{\triangle t}\right)$ & $\triangle t$ & $N_{S}$ \\
\hline \hline 0.8 & 0.92 & $1.5 \mathrm{~ms}$ & 146 \\
0.9 & 0.64 & $1 \mathrm{~ms}$ & 101 \\
0.99 & 0.2 & $0.3 \mathrm{~ms}$ & 31 \\
0.999 & 0.06 & $9.96 e-2 \mathrm{~ms}$ & 10 \\
0.9998 & 0.031 & $5 e-2 \mathrm{~ms}$ & 5 \\
\hline
\end{tabular}

The parameters of the simulation are listed in Table II.

TABLE II

Parameters of THE Simulations.

\begin{tabular}{r|c}
\hline Parameter & Value \\
\hline \hline Number of cells & 3 \\
Number of users per cell & 1 \\
Cell radius & $1 \mathrm{~km}$ \\
Number of Tx antennas per BS & 2 \\
Number of Rx antennas per MS & 2 \\
Distance gain & $G_{P L}=128.1+37.6 \log _{10} d$ \\
Shadow fading standard deviation & $6 \mathrm{~dB}$ \\
Antenna gain & $12 \mathrm{~dB}$ \\
Carrier Frequency & $1.8 \mathrm{GHz}$ \\
System Bandwidth & $100 \mathrm{kHz}$ \\
Rayleigh fading & Yes \\
Users velocity & $60 \mathrm{~km} / \mathrm{h}$ \\
Doppler frequency $f_{D}$ & $100 \mathrm{~Hz}$ \\
Coherence Time & 1 \\
Noise power & $-103 \mathrm{dBm}$ \\
\hline
\end{tabular}

\section{A. Performance Metrics}

In order to evaluated the simulation results, the average spectral efficiency per user $\mathrm{SE}_{\mathrm{avg}}$ is adopted as performance measure. Hence, we need to define the $\operatorname{SINR}_{i}$ of receive antenna $i$ as:

$$
\operatorname{SINR}_{\mathrm{i}}=\frac{\left\|\mathbf{H}_{\mathrm{eq}_{i, i}}\right\|^{2}}{\sum_{j \neq i}|| \mathbf{H}_{\mathrm{eq}_{i, j}}\left\|^{2}+\right\| \mathbf{n} \|^{2}} .
$$

where $\mathbf{H}_{\mathrm{eq}}=\mathbf{H T}$ is the equivalent channel obtained by precoding matrix applied to the channel matrix.

The average spectral efficiency per user is given by:

$$
\mathrm{SE}_{\mathrm{avg}}=\frac{1}{K} \sum_{i=1}^{N_{r} K} \log _{2}\left(1+\mathrm{SINR}_{\mathrm{i}}\right) .
$$

The other performance measurement adopted is the bit error rate (BER), which is calculated considering that the bits are mapped into a QPSK modulation.

\section{B. Results}

Figure 3 compares uncoded BER curves of linear precoding techniques using both perfect and dynamic CSIT with $\rho=$ 0.999 and considering the scenario $(2,2,3,3)$. In low SNRs, the performance obtained with dynamic channel is similar to the perfect channel. In high SNRs, the performance gap between perfect and dynamic increases, mainly with BD precoding. Since that BD was designed to maximize the capacity, it is expected that the BER performance of this technique is worse than the others ones (ZF and MMSE), which have been projected to minimize the average quadratic error.

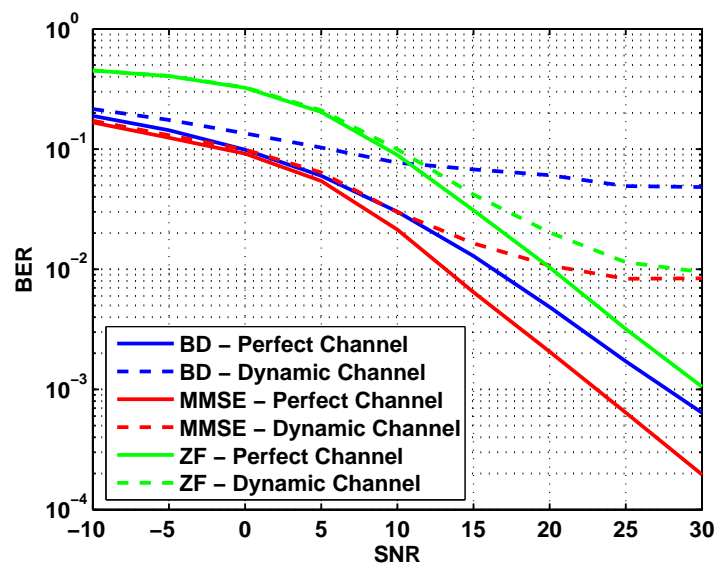

Fig. 3. Uncoded BER curves of precoding techniques using perfect and dynamic channel model $(\rho=0.999)$.

In order to evaluate the behavior of the performance results when the channel temporal variation parameter $\rho$ varies, it is simulated the scenario $(2,2,3,3)$ using MMSE precoding technique and varying the parameter $\rho$ in this scenario. Figures 4 and 5 show the capacity and BER performance, respectively, when the channel temporal correlation increases. Figure 4 shows the capacity obtained with MMSE for high SNRs (in order to have a better view), it is possible to note that the results are similar when the $\rho$ varies. Thus, the capacity reduction is very small with the use of dynamic channel model.

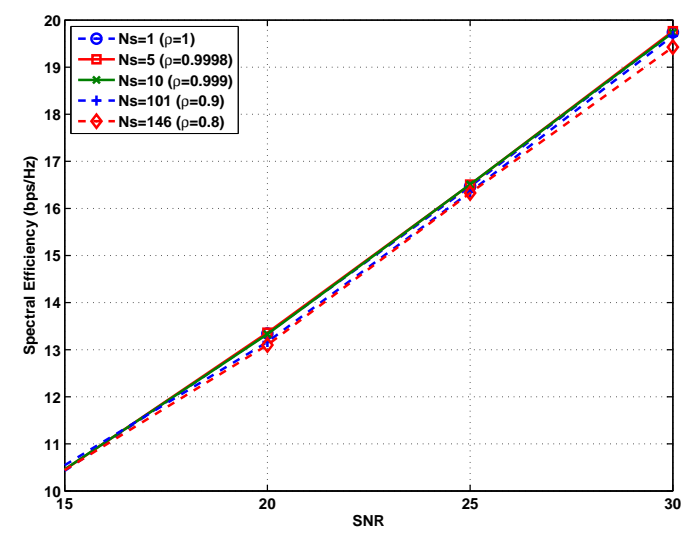

Fig. 4. Capacity curves of MMSE precoding technique using dynamic channel model and varying the channel temporal variation $\rho$.

Figure 5 shows the BER comparison when the parameter $\rho$ increases. It is possible to note that the BER performance is very dependent of $\rho$. The BER curves where $\rho=0.9998$ 
and $\rho=0.999$ show that the results are not bad and, in such cases, the size of channel block is equal to 10 symbols and, in this case, the receiver sends the initial measurement channel $\mathbf{H}_{0}$ to each 10 symbol intervals.

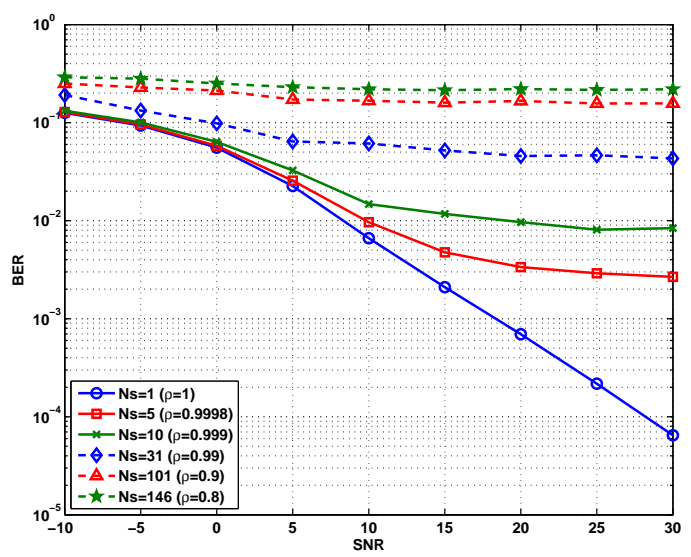

Fig. 5. Uncoded BER curves of MMSE precoding technique using dynamic channel model and varying the channel temporal variation $\rho$.

In the general manner, the performance results improve when the channel temporal variation parameter $\rho$ increases. This is explained because when $\rho$ is equal to 0 the channel estimative coincides with channel mean $\overline{\mathbf{H}}$ and provide less performance gain. On the other hand, when $\rho$ grows toward to 1 , the estimative moves toward the initial channel measurement $\mathbf{H}_{0}$ and the error covariance becomes small (errorfree). Therefore, it is possible to note that the channel temporal variation parameter $\rho$ acts as a channel estimate quality, since that the CSIT ranges from perfect channel knowledge (when $\rho=1$ ) to channel statistics (when $\rho=0$ ).

\section{CONCLUSIONS AND PERspectives}

In this work, the use of linear precoding techniques with CSIT obtained in a dynamic way was evaluated. The scenario considers a multiuser DAS with joint transmission scheme (MCP). This scenario is of great interest due to some advantages, such as, to obtain a good conditioning of the channel matrix and to offer macrodiversity protection for shadowing impairments and also due to considering a heterogenous network and joint transmission scheme.

The multicell multiuser dynamic CSIT model was validated and compared to the case with perfect channel model using the precoders ZF, MMSE and BD. The results showed that the $\mathrm{BD}$ precoding has the most accentuated performance loss. This occurs because BD have been designed not to minimize the BER, but to maximize the capacity. Thus, such technique is sensible to BER when the channel is imperfect.

The influence of the update frequency of the joint initial measurement matrix $\mathbf{H}_{0}$ (increasing the parameter $\rho$ ) in the performance results was evaluated and the results showed that, when the update frequency increases, the performance metrics become better. But, since the increase of the update frequency causes more feedback information, it is necessary to evaluate an equilibrium between performance versus amount of feedback.
A perspective of the work is the investigation of the performance measurements when the number of users per cell is higher than one, since the approximation in equation (17) is guaranteed to be true when there is only one user per cell.

\section{REFERENCES}

[1] H. Hu, Y. Zhang, and J. Luo, Distributed Antenna Systems: Open Architecture for Future Wireless Communications, Auerback Publications, 10 edition, 2007.

[2] G. Dietl and G. Bauch, "Linear precoding in the downlink of limited feedback multiuser mimo," IEEE GLOBECOM'07, pp. 4359-4364, Nov. 2007.

[3] V. Sharma, K. Premkumar, and R.N. Swamy, "Exponential diversity achieving spatio-temporal power allocation scheme for fading channels," IEEE Trans. on Inf. Theory, vol. 54, no. 1, pp. 188-208, Jan. 2008.

[4] M. Kobayashi and G. Caire, "Joint beamforming and scheduling for a multi-antenna downlink with imperfect transmitter channel knowledge," IEEE J. on Sel. Areas in Comm., vol. 25, no. 7, pp. 1468-1477, Sep. 2007.

[5] V. Stankovic and M. Haardt, "Generalized design of multi-user mimo precoding matrices," IEEE Trans. on Wireless Comm., vol. 7, no. 3, pp. 953-961, Mar. 2008.

[6] G. Cinis and J. Cioffi, "A multi-user precoding scheme achieving crosstalk cancellation with application to dsl systems," in in Proc. Asilomar Conf. on Signals, Systems, and Comp., Nov. 2000, vol. 2, pp. 1627-1637.

[7] W. Jakes, Microwave Mobile Communications, Wiley-IEEE Press, Piscataway, NJ, 2nd edition, May 1994.

[8] A. Narula, M.J. Lopez, M.D. Trott, and G.W. Wornell, "Efficient use of side information in multiple-antenna data transmission over fading channels," IEEE J. on Sel. Areas in Comm., vol. 16, no. 8, pp. 14231436, Oct. 1998.

[9] P. Ding, D.J. Love, and M.D. Zoltowski, "Multiple antenna broadcast channels with shape feedback and limited feedback," IEEE Trans. on Sig. Processing, vol. 55, no. 7, pp. 3417-3428, July 2007.

[10] N. Jindal and A. Goldsmith, "Dirty-paper coding versus tdma for mimo broadcast channels," IEEE Trans. on Inf. Theory, vol. 51, no. 5, pp. 1783-1794, May 2005.

[11] D.J. Love, R.W. Heath Jr., and T. Strohme, "Quantized maximum ratio transmission for multiple-input multiple-output wireless system," in Conf. Record of the 36th ACSSC'06, vol. 1, pp. 531-535, Nov. 2002.

[12] K. K. Mukkavilli, A. Sabharwal, E. Erkip, and B. Aazhang, "On beamforming with finite rate feedback in multiple-antenna systems," IEEE Trans. on Inf. Theory, vol. 49, no. 10, pp. 2562-2579, Oct. 2003.

[13] U. Madhow E. Visotsky, "Space-time transmit precoding with imperfect feedback," IEEE Trans. on Inf. Theory, vol. 47, no. 6, pp. 2632-2639, Sept. 2001.

[14] S. A. Jafar, S. Vishwanath, and A. Goldsmith, "Channel capacity and beamforming for multiple transmit and receive antennaswith covariance feedback," in Proc. of IEEE ICC'01, vol. 7, pp. 2266-2270, June 2001.

[15] A. Paulraj M. Vu, "A robust transmit csi framework with applications in mimo wireless precoding," Conference Record of the 39th ACSSC'05., pp. 623-627, Nov. 2005.

[16] A. Paulraj, R. Nabar, and D. Gore, Introduction to Space-Time Wireless Communications, Cambridge University Press, 2003.

[17] C.V. Loan and N. Pitsianis, Approximation with Kronecker Products, Kluwer Publications, 1993.

[18] Q.H. Spencer, A.L. Swindlehurst, and M. Haardt, "Zero-forcing methods for downlink spatial multiplexing in multiuser mimo channels," IEEE Trans. on Sig. Proc., vol. 52, no. 2, pp. 461-471, Feb. 2004.

[19] M. Costa, "Writing on dirty paper (corresp.)," IEEE Trans. on Inf. Theory, vol. 29, no. 3, pp. 439-441, May 1983.

[20] C. Peel, B. Hochwald, and L. Swindlehurst, "A vector-perturbation technique for near-capacity multi-antenna multi-user communication," in In Proc. of the 41st Allerton Conf. on Comm., Control, and Computing., Oct. 2003.

[21] C. Windpassinger, R. F. H. Fischer, and J. B. Huber, "Lattice-reductionaided broadcast precoding," in in Proc. 5th International ITG Conf. on Source and Channel Cod., Erlangen, Germany, Jan. 2004, pp. 403-408.

[22] H. Zhang and H. Dai, "Cochannel interference mitigation and cooperative processing in downlink multicell multiuser mimo networks," EURASIP J. on Wireless Comm. and Net., , no. 2, pp. 222-235, July 2004.

[23] M. Joham, W. Utschick, and J.A. Nossek, "Linear transmit processing in mimo communications systems," IEEE Trans. on Sig. Proc., vol. 53, no. 8, pp. 2700-2712, Aug. 2005 . 